\title{
BIOAVAILABILITY AND DISSOLUTION ENHANCEMENT OF GLYBURIDE NANOSUSPENSION
}

\author{
PRADEEP KUMAR M*, CHANDRA SEKHAR KB
}

Department of Pharmaceutical Sciences, Jawaharlal Nehru Technological University, Ananthapuram, Andhra Pradesh, India. Email: pradeepbadvel98@gmail.com

Received: 22 January 2019, Revised and Accepted: 19 February 2019

ABSTRACT

Objective: The main objective was to develop nanosuspension of glyburide (GLY) by quasi emulsification solvent diffusion method and to enhance dissolution and bioavailability characteristics of the drug GLY, an antidiabetic drug which belongs to Biopharmaceutical Classification System-II category.

Methods: In this work, nanoparticles were prepared using polyvinyl alcohol, hydroxypropyl methyl cellulose, and Eudragit RL100. Twelve formulations of GLY (GLY-1-GLY-12) were formulated using the excipients at various compositions. Drug and excipient compatibility studies were conducted using Fourier transform infrared and differential scanning calorimeter. The prepared nanosuspension was analyzed using scanning electron microscopy for surface of the particle analysis, melting point, solubility, particle charge zeta ( $m v)$, percentage drug entrapment efficiency (\%), and in vitro drug release. The optimized formulations of nanosuspension were further studied for in vivo pharmacokinetic evaluation. Reverse-phase high-performance liquid chromatography method was developed, validated, and used for the study of these formulations in rat plasma.

Results: From these studies, it was confirmed that drugs and excipients chosen were compatible with each other. GLY-8 was the best formulation with a particle size of $85-96 \mathrm{~nm}$ with $168.7^{\circ} \mathrm{C}$ melting point, freely soluble in phosphate buffer $\mathrm{pH} 7.4,93.53 \%$ drug entrapment, and $90.26 \pm 1 \mathrm{mV}$ of zeta potential. This formulation shows percentage drug release of $99.85 \%$ in $24 \mathrm{~h}$. In vivo pharmacokinetic study for optimized formulation (GLY -8) suggested that there was no reaction with the rat plasma. From the results, it was shown that $\mathrm{C}_{\max }$ and $\mathrm{T}_{\max }$ were found to be $0.604 \pm 0.03 \mu \mathrm{g} / \mathrm{ml}$ and $2 \pm 1.01 \mathrm{~h}$, respectively. The values of $\mathrm{t}_{1 / 2}(\mathrm{~h})$, area under the curve $(\mathrm{AUC})_{(0-\mathrm{t})}$, and AUC ${ }_{(0-\infty)}$ were found to be $10.04 \mathrm{~h}, 2.562 \pm 0.41 \mu \mathrm{g} . \mathrm{h} / \mathrm{ml}$, and $2.147 \pm 0.45 \mu \mathrm{g} . \mathrm{h} / \mathrm{ml}$, respectively.

Conclusion: Based on the results obtained, oral administration of nanosuspension could not only provide the better absorption of poorly water soluble drugs but may also reduce toxicity and provide a new tool in drug delivery system.

Keywords: Glyburide, Scanning electron microscopy studies, In vitro drug release, In vivo pharmacokinetic evaluation, Dissolution and bioavailability enhancement.

(C) 2019 The Authors. Published by Innovare Academic Sciences Pvt Ltd. This is an open access article under the CC BY license (http://creativecommons. org/licenses/by/4. 0/) DOI: http://dx.doi.org/10.22159/ajpcr.2019.v12i4.31657

\section{INTRODUCTION}

In the drug discovery and development, poor water solubility is regarded as wide problem. More than $40 \%$ of drugs are poorly soluble in water, so they show problems in formulating them in conventional dosage forms. This problem is complex for Class II drugs which are poorly soluble in aqueous and organic media. Nanosuspension preparation is preferred for compounds that are insoluble in water (but are soluble in oil) with high $\log \mathrm{p}$ value. Various approaches are there to resolve problems of low solubility and low bioavailability such as micronization, cosolvency, oily solution, and salt formation, and some other techniques are liposomes, emulsions, microemulsion, solid dispersion, and $\beta$-cyclodextrins inclusion complex. In these cases, nanosuspensions are preferred. It is most suitable for the compounds with high log $\mathrm{p}$ value, high melting point, and high dose. Nanosuspensions can be used to enhance the solubility of drugs that are poorly soluble in aqueous as well as lipid media. As a result, the rate of flooding of the active compound increases and the maximum plasma level is reached faster (e.g., oral or intravenous administration of the nanosuspension). This is one of the unique advantages that it has over other approaches for enhancing solubility. It is useful for molecules with poor solubility, poor permeability, or both, which poses a significant challenge for the formulators [1]. Glibenclamide which is also known as glyburide (GLY) belongs to second-generation sulfonylurea. It is majorly used to treat noninsulin-dependent diabetes mellitus and administered orally [2]. GLY has been classified as the Biopharmaceutical Classification System (BCS) class-II drug due to its low aqueous solubility, with bioavailability of $100 \%$ and huge permeability. The solubility of GLY in gastrointestinal fluids and $\mathrm{pH}$ in the gastrointestinal tract impact on its in vivo dissolution. A lot of approaches have been made to optimize in vivo dissolution and bioavailability. However, to predict the in vivo performance of a dosage form, it is necessary to have an in vitro-in vivo correlation studies. The main objective of this study was to develop nanosuspension of GLY by quasi emulsification solvent diffusion method and also to enhance dissolution and bioavailability. This manuscript includes 12 formulations of GLY which were prepared with three different polymers such as polyvinyl alcohol (PVA), hydroxypropyl methyl cellulose (HPMC), and Eduragit (EDG) with a stabilizer, i.e. poloxamer 407 with formulation codes from GLY-1 to GLY-12. The best formulation had been identified and further subjected to both in vitro and in vivo studies [3-5]. The present study brought out the best formulation with an enhanced dissolution and bioavailability.

\section{MATERIALS AND METHODS}

\section{Materials}

GLY drug sample was procured from Hetero Drugs, Hyderabad. HPMC was procured from Yucca Enterprises, Mumbai. PVA was brought from Yarrow Chem Products., Mumbai. EDG RL 100 was procured from Yucca Enterprises, Mumbai. Poloxamer 407 was procured from SD Fine Chem, Hyderabad. All other chemicals and reagents utilized in the study were with the analytical grade.

\section{Analytical method development}

Determination of $\lambda_{\max }$

Ultraviolet (UV) spectrum of GLY was carried out in phosphate buffer pH 7.4.10 mg of GLY was weighed accurately and transferred to a $10 \mathrm{ml}$ 
volumetric flask. The solution of $10 \mu \mathrm{g} / \mathrm{ml}$ was kept in a fused silica cuvette. UV spectrum was recorded in the range of 200-800 nm by Shimadzu double-beam UV-visible spectrophotometer against blank buffer solution $\mathrm{pH}$ 7.4. The wavelength for maximum absorbance was recorded [6].

\section{Preparation of stock solution}

Accurately weighed $10 \mathrm{mg}$ of GLY was dissolved in $100 \mathrm{ml}$ of phosphate buffer pH 7.4 to get a concentration of $100 \mu \mathrm{g} / \mathrm{ml}$.

\section{Preparation of standard solution}

From the above stock solution, 1, 2, 3, 4, and $5 \mathrm{ml}$ were transferred to a $10 \mathrm{ml}$ volumetric flask and volume was made up to the mark with phosphate buffer $\mathrm{pH} 7.4$. The final concentrations of $10,20,30,40$, and $50 \mu \mathrm{g} / \mathrm{ml}$ were obtained. Absorbance of the above standard solutions was determined in a UV-visible spectrophotometer and calibration curve (CC) was constructed between concentration and absorbance [7].

\section{Fourier transform infrared (FTIR) spectroscopic analysis}

FTIR spectra of GLY were studied. GLY and KBR were mixed and compressed into a pellet in the ratio of 1:100 in a motorized pellet press (Ki Maya Engineers, India) at 10:12 tons of pressure. The pressed pellet was scanned in FTIR spectrophotometer. The FTIR spectrum of GLY was compared with the standard pharmacopoeial spectrum of GLY [8].

\section{Preparation of GLY nanosuspension}

GLY nanosuspensions were prepared by quasi emulsification solvent diffusion method. GLY (30 mg), PVP, HPMC, and EDG RL100 (0.5, 1.0, 1.5 , and $2 \%$ ) were codissolved in $15 \mathrm{ml}$ of methanol. The solution was slowly injected with a syringe containing thin Teflon tube into $35 \mathrm{ml}$ water containing poloxamer 407. It was maintained at low temperature in ice bath to protect from sun light. During injection, the mixture was stirred well by a high-speed homogenizer at a speed of $5500 \mathrm{rpm}$. The solution immediately turned into pseudo emulsion in the external aqueous phase. The counter diffusion of methanol and water out of and into the emulsion microdroplets, respectively, results into the formation of nanosuspension. Formulation was prepared with varying polymer ratio. 12 formulations of GLY were prepared with three different polymers such as PVA, HPMC, and EDG with a stabilizer, i.e., poloxamer 407 with formulation codes from GLY-1 to GLY-12. Table 1 gives the formulation of GLY nanosuspensions [9].

\section{Physicochemical evaluation of drugs}

Color and appearance

The color and appearance of drug were observed and recorded.

\section{Melting point}

The melting point of GLY was determined by open capillary method. The melting point was determined by introducing small amount of substance in the capillary which was then attached to graduated thermometer.
Later, constant heat was applied with the assembly suspended in the paraffin bath. The drug sample was tested at the temperature ranging between 200 and $250^{\circ} \mathrm{C}$. The temperature required to melt drug was noted.

\section{Solubility studies}

Solubility studies were done by adding $100 \mathrm{mg}$ of drug in increments to $10 \mathrm{ml}$ of different buffer solutions. The conical flasks with $250 \mathrm{ml}$ of water were kept on a shaker water bath until saturation occurred. The conical flasks were kept for $5 \mathrm{~h}$ at ambient temperature. The samples were centrifuged and filtered and the filtrates were analyzed by UVvisible spectrophotometer [10]

\section{Evaluation of GLY nanosuspension}

Size analysis

Scanning electron microscopy (SEM) is a method for high-resolution surface imaging. The SEM uses an electron beam for surface imaging. The advantages of SEM over light microscopy are greater magnification and much larger depth of field. Different elements and surface topographies emit different quantities of electrons, due to which the contrast in a SEM micrograph (picture) is representative of the surface topography and distribution of elemental composition on the surface [11].

\section{Zeta potential of the drug}

Zeta potential measurements were run at $25^{\circ} \mathrm{C}$ with an electric field strength of $23 \mathrm{~V} / \mathrm{m}$, using Zetasizer (Nano ZS 90, Malvern Instruments, UK). To determine the zeta potential, samples of drug nanosuspensions were diluted and placed in electrophoretic cell. The zeta potential was calculated as described by Helmholtz-Smoluchowski equation. The formulation of GLY with PVA, HPCM, and EDG RL 100 with a stabilizer poloxamer 407 (Pluronic F127) was done. After formulating the suspension or nanosuspension, the zeta potential was determined to know the stability of the nanosuspension. Zeta potential is very important as it measures stability. Hence, all formulations were determined with zeta potential and recorded [12].

\section{Drug content}

$$
\% \text { Drug content }=\frac{\text { Obtainedamount of drug }}{\text { Theoratical amount of drug }}
$$

A weighed amount of each preparation was dissolved in the required amount of methanol and diluted suitably in phosphate buffer of $\mathrm{pH}$ 7.4. Spectrophotometrically, drug content was determined at required wavelength. Calculation was done using the following formula [13].

\section{Percentage entrapment efficiency}

To determine percentage entrapment, around $2 \mathrm{ml}$ of each formulation was taken in $\mathrm{c}(10 \mathrm{ml})$ and was centrifuged in centrifuge machine at 2000-3000 rpm for $4 \mathrm{~h}$. The supernatant layer was filtered through Whatman filter paper no: 41 and diluted with phosphate buffer

Table 1: Formulation of GLY nanosuspensions using emulsification solvent diffusion method

\begin{tabular}{|c|c|c|c|c|c|c|}
\hline Formula (mg) & GLY & PVA (\%) & HPMC (\%) & EDG & Poloxamer (mg) & Distilled water $(\mathrm{ml})$ \\
\hline GLY-1 & 30 & 0.5 & - & - & 200 & 50 \\
\hline GLY-2 & 30 & 1.0 & & & 200 & 50 \\
\hline GLY-3 & 30 & 1.5 & & & 200 & 50 \\
\hline GLY-4 & 30 & 2.0 & & & 200 & 50 \\
\hline GLY-5 & 30 & & 0.5 & & 200 & 50 \\
\hline GLY-6 & 30 & & 1.0 & & 200 & 50 \\
\hline GLY-7 & 30 & & 1.5 & & 200 & 50 \\
\hline GLY-9 & 30 & & & 0.5 & 200 & 50 \\
\hline GLY-10 & 30 & & & 1.0 & 200 & 50 \\
\hline GLY-11 & 30 & & & 1.5 & 200 & 50 \\
\hline GLY-12 & 30 & & & 2.0 & 200 & 50 \\
\hline
\end{tabular}

GLY: Glyburide, PVA: Polyvinyl alcohol, HPMC: Hydroxypropyl methyl cellulose, EDG: Eudragit 
$\mathrm{pH}$ 7.4. The resultant solutions were analyzed at a wavelength of $\mathrm{nm}$ of respective drug using UV double-beam spectrophotometer. These readings were taken for 3 times and the result was calculated [14]. The percentage entrapment efficiency was calculated according to the following equation or formula:

$\mathrm{EE}=($ Total drug content - free drug content/Total drug content $) \times 100$

\section{Differential scanning calorimetry (DSC)}

GLY thermal behavior was assessed by carrying out thermal analysis by DSC (DSC-Hitachi 7020). The samples (8-10 mg) were carefully transferred and heated in a crimped aluminum pan for accurate results. The samples were heated from 100 to $600^{\circ} \mathrm{C}$ at the rate of $10^{\circ} \mathrm{C} / \mathrm{min}$. A physical mixture of GLY with polymers (PVA, HPMC, and EDG) in the ratio of $1: 1$ was assessed by carrying out thermal analysis. Thermogram for drug and polymer mixture was taken by using DSC (Mettler DSC 1 star system, Mettler-Toledo, Switzerland) [15].

\section{In vitro drug release studies}

In vitro drug release of the nanosuspension was carried out using USP dissolution apparatus type 2 (paddle type). $5 \mathrm{ml}$ of nanosuspension was taken in a dialysis membrane consisting of spectra or membrane (cutoff: $1200 \mathrm{Da}$ ). This dialysis system was tied to paddle and dissolution medium with phosphate buffer $\mathrm{pH}$ 7.4. Dissolution was

Table 2: CC data of GLY in phosphate buffer pH 7.4

\begin{tabular}{lll}
\hline Sl. No & Concentration $(\mu \mathrm{g} / \mathbf{m l})$ & Absorbance at $\mathbf{2 2 9} \mathbf{~ n m}$ \\
\hline 1 & 25 & 0.298 \\
2 & 50 & 0.440 \\
3 & 75 & 0.602 \\
4 & 100 & 0.732 \\
5 & 125 & 0.869 \\
6 & 150 & 0.992 \\
\hline
\end{tabular}

GLY: Glyburide, CC: Calibration curve

Table 3: Physical properties of drug

\begin{tabular}{lll}
\hline Sl. No & Parameter & Drug (GLY) \\
\hline 1 & Color & White \\
2 & Appearance & Crystalline \\
\hline GLY: Glyburide & &
\end{tabular}

Table 4: Melting point determination of drug

\begin{tabular}{ll}
\hline Drug name & GLY $^{\circ} \mathbf{C}$ \\
\hline Reported melting point & $165-171$ \\
Observed Melting Point & 168.7 \\
\hline
\end{tabular}

carried in triplicate for $10 \mathrm{~h}$ at $37 \pm 10^{\circ} \mathrm{C}$ temperature and $50 \mathrm{rpm}$. At regular intervals of time, $1 \mathrm{ml}$ of sample from the external medium was taken and replaced with fresh phosphate buffer and all the samples were analyzed at $\mathrm{nm}$ of respective drug using U.V spectrophotometer. GLY nanosuspensions were prepared by quasi emulsification method which were analysed by in vitro drug dissolution method $[7,11]$.

\section{Mathematical dissolution model for GLY nanosuspension formulations}

Various mathematical dissolution models such as Higuchi, KrosmeyerPeppas, and first order were applied for GLY nanosuspension to evaluate the release kinetics of GLY nanosuspension.

\section{In vivo studies}

Bioavailability studies in Wistar rats

Male Wistar rats weighing about $200 \pm 20 \mathrm{~g}$ were supplied by the Experimental Animal Center of Local Vendor, and animal experiment was evaluated and approved by the Institution of Animal Ethics Committee (1423/PO/a/04/CPCSEA/104/2018). Rats were randomly divided into two groups with six animals each and were caged at least for 3 days before the study. They had free access to both food and water. Food was withdrawn $12 \mathrm{~h}$ before start of the study. An optimized formulation of nanosuspension was administrated orally to rats [16].

\section{RESULTS}

\section{Analytical method for GLY}

Determination of $\lambda_{\max }$

UV spectra of GLY in phosphate buffer $\mathrm{pH} 7.4$ were determined by double beam UV-Visible spectrophotometer and found to be at $229 \mathrm{~nm}$. Fig. 1 shows the $\lambda_{\max }$ curve of GLY.

\section{CC and physical properties of GLY}

CC of GLY was constructed by determining the absorbance of GLY at $229 \mathrm{~nm}$. It was observed that GLY showed good linearity over the concentration range of $25-150 \mu \mathrm{g} / \mathrm{ml}$. Table 2 and Fig. 2 show calibration and linearity curve data of GLY, respectively. The color of GLY was white and crystalline in appearance Table 3.

\section{Drug-excipient compatibility studies}

Drug and excipient compatibility studies were performed using FTIR spectrophotometer and DSC, and no marked incompatibilities were found which was shown in Fig 3 and 4.

\section{Physical properties of GLY nanosuspension Melting point}

Capillary tube method was used to determine melting point and was found to be $168.7^{\circ} \mathrm{C}, \mathrm{GLY}$. This value is the same as that of the literature citation shown in Table 4

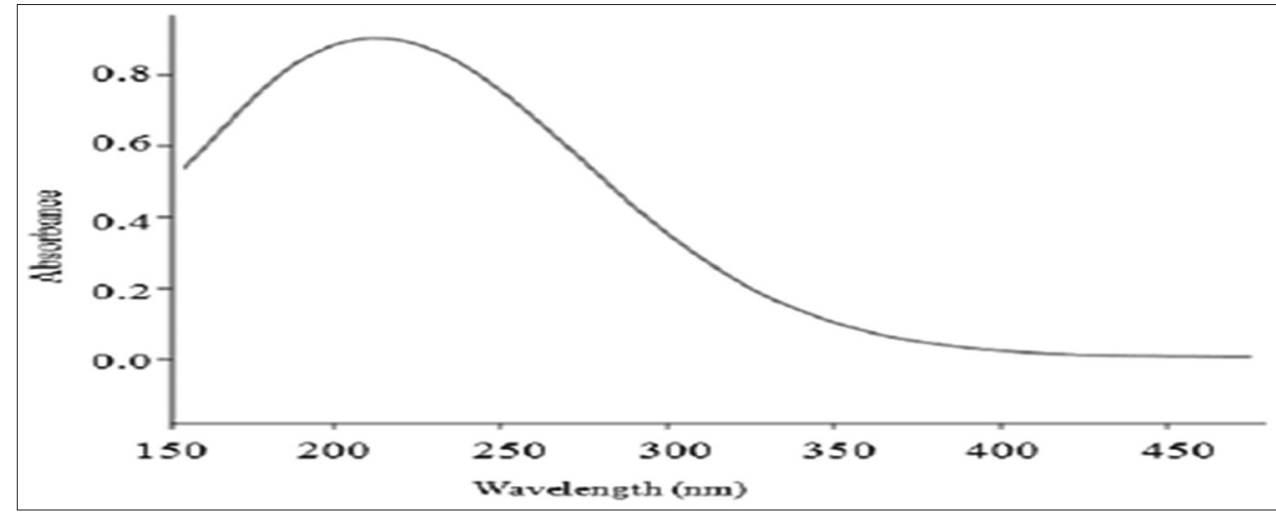

Fig. 1: $\lambda_{\max }$ curve of glyburide in phosphate buffer $\mathrm{pH} 7.4$ 
Solubility studies

Table 5 shows the results of solubility studies according to pharmacopeia limits

\section{Evaluation of GLY nanosuspension}

SEM

SEM of optimized formula (GLY-8) is shown in Fig. 5 and SEM analysis of different formulations is described in Table 6.

\section{DSC}

DSC thermograms of pure GLY and GLY plus polymer blend were mentioned in Figs. 6 and 7.

In vitro drug release studies: Table 7 and Fig 8 showed the percentage cumulative drug release of various GLY nanosuspensions.

Mathematical dissolution model for GLY nanosuspension formulations

Variousmathematicaldissolution models suchasHiguchi,Korsmeyer-Peppas, and first order were applied for GLY nanosuspension to evaluate the release kinetics of GLY nanosuspension. Table 8 and Figs. 9-11 give the data and plots of Higuchi, Krosmeyer-Peppas, and first order, respectively.

\section{Table 5: Solubility studies}

\begin{tabular}{ll}
\hline Medium & $\mathbf{m g} / \mathbf{1 0 0} \mathbf{~ m l}$ \\
\hline Water & Insoluble \\
Phosphate buffer pH (6.8) & Very slightly soluble \\
Methanol & Soluble \\
Chloroform $\left(\mathrm{CHCL}_{3}\right)$ & Soluble \\
\hline
\end{tabular}

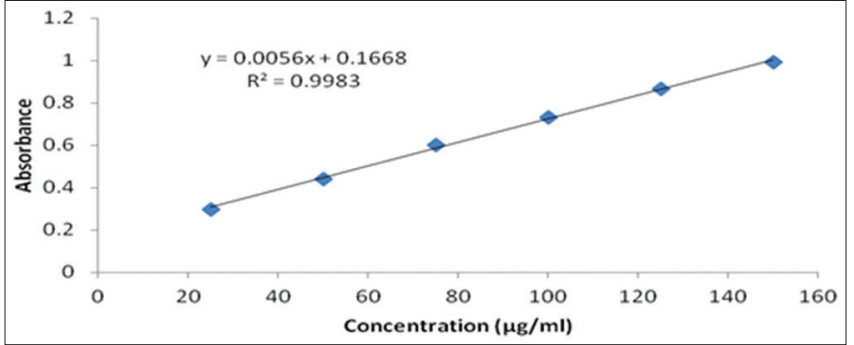

Fig. 2: Calibration curve of glyburide in phosphate buffer pH 7.4

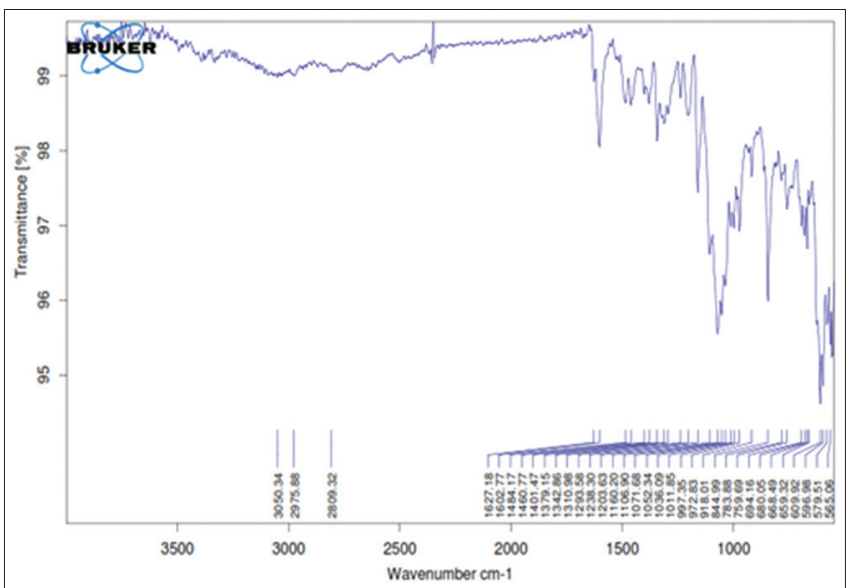

Fig. 3: Fourier transformed infrared spectrum of glyburide

Table 6: Evaluation studies of particle size (nm), drug content, \% drug entrapped, and zeta potential (mV) for GLY nanosuspension

\begin{tabular}{llllll}
\hline S. No & Formulation & Particle size & Drug content & \% Drug entrapped & Zeta potential \\
\hline 1 & GLY-1 & $198-225$ & $78.62 \pm 0.24$ & 77.11 & $61.58 \pm 1$ \\
2 & GLY-2 & $196-210$ & $96.38 \pm 0.84$ & 75.90 & $65.20 \pm 1$ \\
3 & GLY-3 & $215-230$ & $87.47 \pm 1.41$ & 71.89 & $70.15 \pm 2$ \\
4 & GLY-4 & $241-236$ & $75.23 \pm 0.59$ & 80.75 & $79.55 \pm 2$ \\
5 & GLY-5 & $152-163$ & $79.66 \pm 0.45$ & 89.48 & $81.48 \pm 2$ \\
6 & GLY-6 & $172-189$ & $92.84 \pm 0.57$ & 88.80 & $85.22 \pm 1$ \\
7 & GLY-7 & $111-125$ & $88.45 \pm 1.22$ & 84.76 & $90.26 \pm 1$ \\
8 & GLY-8 & $85-96$ & $98.93 \pm 0.62$ & 93.53 & $35.10 \pm 2$ \\
9 & GLY-9 & $325-315$ & $78.21 \pm 0.47$ & 57.52 & $31.59 \pm 1$ \\
10 & GLY-10 & $354-385$ & $85.56 \pm 0.81$ & 60.76 & $33.45 \pm 1$ \\
11 & GLY-11 & $321-361$ & $83.26 \pm 0.66$ & 58.75 & 61.16 \\
12 & GLY-12 & $311-320$ & $92.47 \pm 1.12$ & & 3.15 \\
\hline
\end{tabular}

Table 7: Percentage cumulative drug release data of glyburide nanosuspension

\begin{tabular}{|c|c|c|c|c|c|c|c|c|c|c|c|c|}
\hline \multirow[t]{2}{*}{ Time (H) } & \multicolumn{12}{|c|}{ Percentage cumulative drug release } \\
\hline & GLY-1 & GLY-2 & GLY-3 & GLY-4 & GLY-5 & GLY-6 & GLY-7 & GLY-8 & GLY-9 & GLY-10 & GLY-11 & GLY-12 \\
\hline 0 & 0 & 0 & 0 & 0 & 0 & 0 & 0 & 0 & 0 & 0 & 0 & 0 \\
\hline 1 & 18.26 & 15.8 & 13.44 & 10.22 & 17.55 & 15.22 & 13.22 & 8.22 & 18.22 & 15.25 & 13.85 & 10.43 \\
\hline 2 & 31.52 & 22.84 & 23.54 & 19.52 & 28.65 & 24.65 & 25.36 & 18.65 & 34.25 & 24.65 & 20.65 & 24.36 \\
\hline 3 & 49.55 & 38.41 & 35.41 & 28.47 & 40.01 & 35.65 & 34.58 & 24.15 & 50.22 & 34.22 & 28.69 & 32.84 \\
\hline 4 & 61.2 & 55.74 & 42.68 & 37.85 & 59.14 & 48.52 & 47.26 & 30.17 & 68.24 & 47.15 & 35.01 & 40.21 \\
\hline 5 & 78.55 & 69.14 & 54.21 & 46.24 & 72.36 & 60.2 & 55.3 & 39.98 & 79.25 & 56.84 & 42.59 & 48.65 \\
\hline 6 & 88.49 & 80.14 & 68.11 & 57.15 & 88.24 & 78.52 & 65.84 & 45.21 & 89.22 & 61.47 & 50.22 & 57.1 \\
\hline 7 & - & 99.16 & 78.1 & 65.85 & 99.28 & 86.21 & 74.22 & 52.02 & - & 75.26 & 65.64 & 65.95 \\
\hline 8 & - & - & 85.68 & 78.21 & - & 98.52 & 84.62 & 64.22 & - & 86.22 & 75.21 & 72.8 \\
\hline 9 & - & - & 97.02 & 85.25 & - & - & 90.11 & 70.84 & - & 98.14 & 88.89 & 80.41 \\
\hline 10 & - & - & - & 97.11 & - & - & 99.45 & 81.58 & - & - & 99.45 & 89.79 \\
\hline 11 & - & - & - & - & - & - & - & 90.24 & - & - & - & 98.02 \\
\hline 12 & - & - & - & - & - & - & - & 99.85 & - & - & - & - \\
\hline
\end{tabular}

GLY: Glyburide 
In vivo pharmacokinetic studies

Determination of GLY in rat $K_{2}$ ethylenediaminetetraacetic acid plasma

Preparation of CC standards

The stock solutions ranging from $0.1 \mu \mathrm{g} / \mathrm{ml}$ to $1.0 \mu \mathrm{g} / \mathrm{ml}$ were prepared by diluting with mobile phase from main stock solution. Table 9 and Fig. 12 show CC data of GLY in rat plasma.

\section{Evaluation of In vivo pharmacokinetic parameters}

Based on in vitro drug release studies, GLY-8 formulation was selected for further in vivo pharmacokinetic characterization in rats.

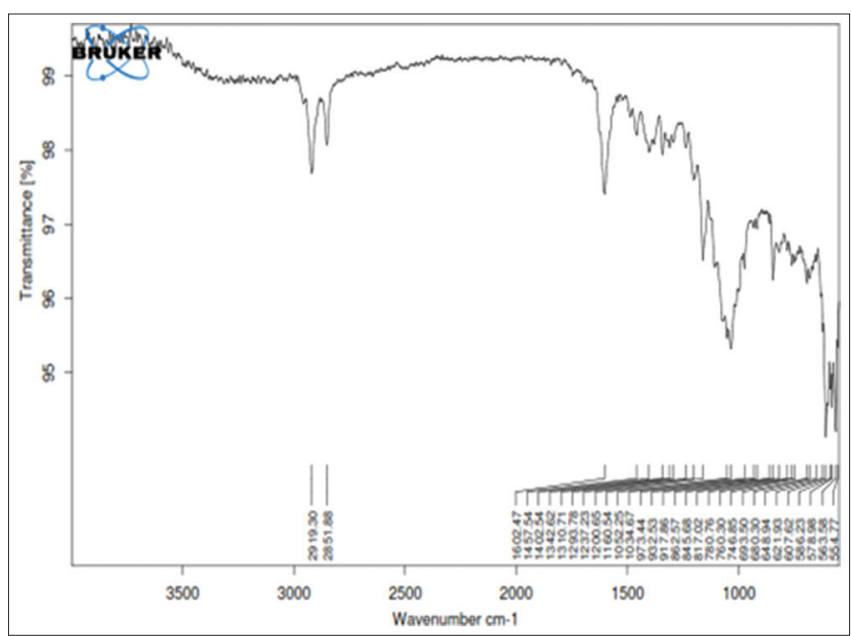

Fig. 4: Fourier transformed infrared spectrum of glyburide plus polymer blend

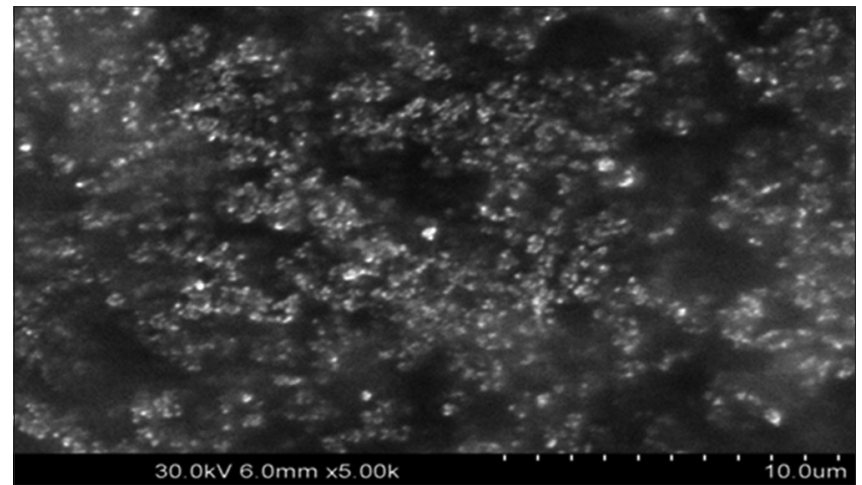

Fig. 5: Scanning electron microscopy photograph of glyburide-8 nanosuspension

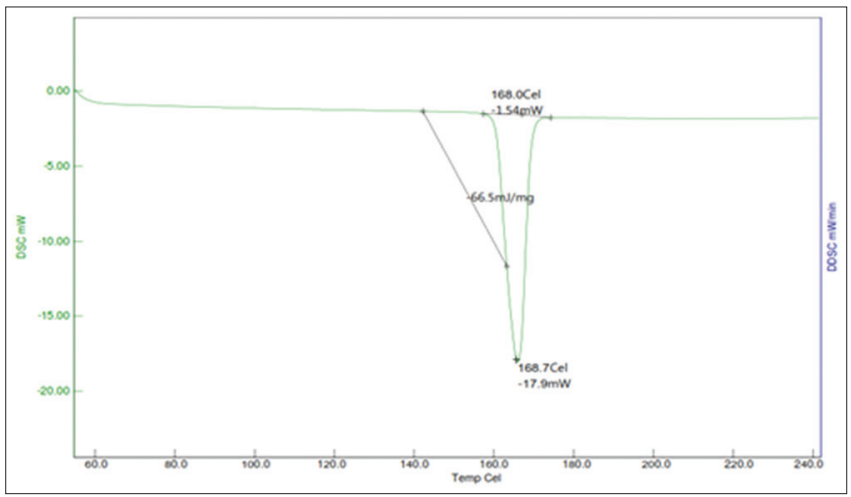

Fig. 6: Differential scanning calorimetry thermogram of pure glyburide
Plasma concentration of GLY in rats $(1 \mathrm{mg} / \mathrm{kg}$ body weight [b.w.], p.o) is presented in the table. Table 10 summarizes the mean plasma concentration of GLY in rats (1 mg/kg b.w., p.o,) which was considered for computation of pharmacokinetic parameters. Figs. 13 and 14 show the representative chromatogram of GLY from plasma samples.

$\mathrm{C}_{\text {max }}$ : Peak plasma concentration attained by GLY (GLY) was $0.604 \pm 0.03 \mu \mathrm{g} / \mathrm{ml}$ following per oral administration.

$\mathrm{T}_{\text {max }}$ : Time required for attaining peak plasma concentration by GLY (GLY), following per oral administration, was $2 \pm 1.01 \mathrm{~h}$.

Area under the curve (AUC): $\mathrm{AUC}_{(0-\mathrm{t})}$ was calculated, and it was found to be $2.562 \pm 0.41 \mu \mathrm{g} . \mathrm{h} / \mathrm{ml}$ for GLY. AUC ${ }_{(0-\infty)}$ was calculated, and it was found to be $2.147 \pm 0.45 \mu \mathrm{g} / \mathrm{ml}$ for GLY.

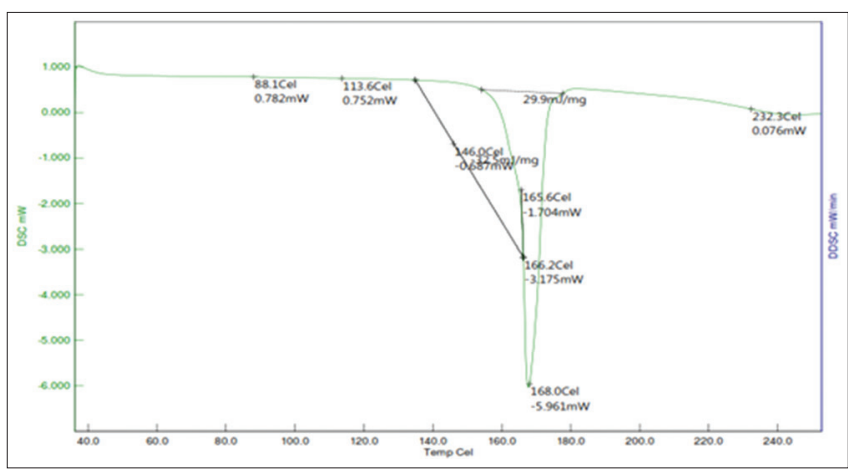

Fig. 7: Differential scanning calorimetry thermogram of glyburide plus polymer blend

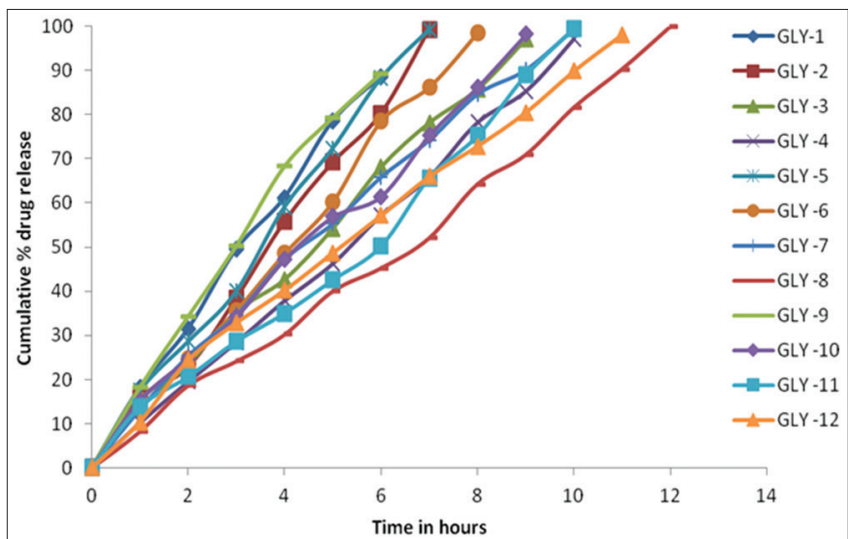

Fig. 8: Percentage cumulative drug release from glyburide nanosuspension

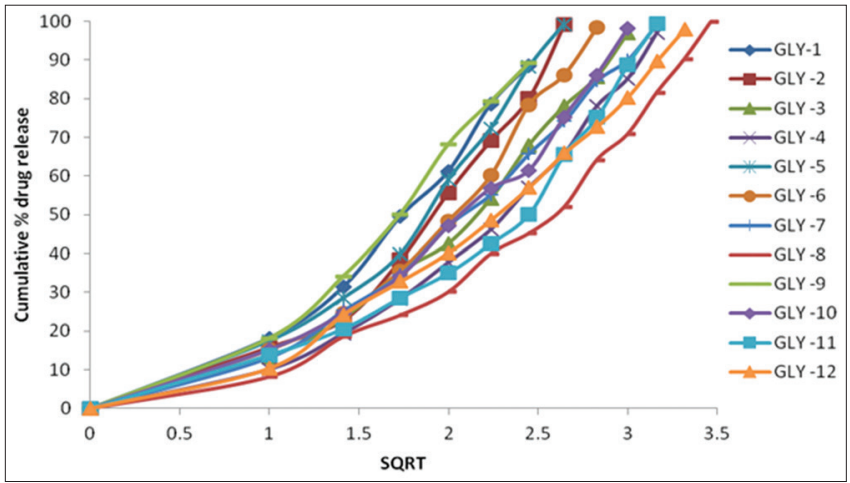

Fig. 9: Higuchi plot 
Table 8: Kinetic correlation coefficients and diffusion exponent data of various kinetic models of GLY nanosuspension

\begin{tabular}{|c|c|c|c|c|c|}
\hline \multirow[t]{2}{*}{ Formulation code } & \multicolumn{4}{|c|}{ Correlation coefficient values $\left(R^{2}\right)$} & \multirow[t]{2}{*}{ Diffusion exponent value (n) } \\
\hline & Zero order & Higuchi's model & Korsmeyer-Peppas & First order & \\
\hline GLY-1 & 0.997841 & 0.965893 & 0.900845 & -0.134 & 0.900766 \\
\hline GLY-2 & 0.997247 & 0.944732 & 0.980874 & -0.13393 & 0.980738 \\
\hline GLY-3 & 0.998848 & 0.962434 & 0.9099 & -0.10033 & 0.909931 \\
\hline GLY-4 & 0.9995 & 0.955341 & 0.978083 & -0.09885 & 0.978057 \\
\hline GLY-5 & 0.99842 & 0.955166 & 0.921243 & -0.12429 & 0.921226 \\
\hline GLY-6 & 0.998244 & 0.95406 & 0.927203 & -0.11286 & 0.92719 \\
\hline GLY-7 & 0.996914 & 0.974591 & 0.874915 & -0.08667 & 0.874964 \\
\hline GLY-8 & 0.997 & 0.950947 & 0.975553 & -0.08486 & 0.975513 \\
\hline GLY-9 & 0.995607 & 0.970394 & 0.905735 & -0.13314 & 0.905721 \\
\hline GLY-10 & 0.997745 & 0.964084 & 0.85161 & -0.09417 & 0.851576 \\
\hline GLY-11 & 0.993451 & 0.94033 & 0.870733 & -0.09121 & 0.870795 \\
\hline GLY-12 & 0.997648 & 0.97353 & 0.890978 & -0.08182 & 0.890924 \\
\hline
\end{tabular}

GLY: Glyburide

Table 9: CC data of GLY in rat plasma

\begin{tabular}{|c|c|c|c|c|c|c|}
\hline Concentration $(\mu \mathrm{g} / \mathrm{ml})$ & Peak area 1 & Peak area 2 & Peak area 3 & Mean Peak area & Standard Deviation & $\%$ RSD \\
\hline 0.1 & 659782 & 652134 & 659423 & 657113 & 4315.68 & 0.66 \\
\hline 0.2 & 1058864 & 1037138 & 1060168 & 1052057 & 12936.39 & 1.23 \\
\hline 0.4 & 1313316 & 1330325 & 1326036 & 1323226 & 8845.90 & 0.67 \\
\hline 0.6 & 1706481 & 1735033 & 1709901 & 1717138 & 15591.29 & 0.91 \\
\hline 0.8 & 1950506 & 1941450 & 1940379 & 1944112 & 5563.49 & 0.29 \\
\hline 1.0 & 2251880 & 2216008 & 2213456 & 2227115 & 21485.33 & 0.96 \\
\hline
\end{tabular}

CC: Calibration curve, GLY: Glyburide

Table 10: Mean plasma concentration of GLY following oral administration

\begin{tabular}{|c|c|}
\hline Time point (h) & $\begin{array}{l}\text { Concentration } \pm \text { standard } \\
\text { deviation }(\mu \mathrm{g} / \mathrm{ml})\end{array}$ \\
\hline 0 & $0.200 \pm 0.05$ \\
\hline 0.5 & $0.369 \pm 0.06$ \\
\hline 1 & $0.440 \pm 0.07$ \\
\hline 2 & $0.604 \pm 0.03$ \\
\hline 4 & $0.524 \pm 0.04$ \\
\hline 6 & $0.316 \pm 0.05$ \\
\hline 8 & $0.119 \pm 0.05$ \\
\hline 10 & $0.007 \pm 0.01$ \\
\hline 12 & $0.200 \pm 0.01$ \\
\hline $\mathrm{C}_{\max }(\mu \mathrm{g} / \mathrm{ml})$ & $0.604 \pm 0.03$ \\
\hline $\mathrm{T}_{\max }(\mathrm{h})$ & $2 \pm 1.01$ \\
\hline $\mathrm{AUC}_{(0-\mathrm{t})}(\mu \mathrm{g} \cdot \mathrm{h} / \mathrm{ml})$ & $2.562 \pm 0.41$ \\
\hline $\operatorname{AUC}_{(0-\infty)}^{(0-\infty)}(\mu \mathrm{g} \cdot \mathrm{h} / \mathrm{ml})$ & $2.147 \pm 0.45$ \\
\hline $\mathrm{T}_{1 / 2}(\mathrm{~h})$ & 10.04 \\
\hline
\end{tabular}

$\mathrm{T}_{1 / 2}$ : Time required for a drug to decrease by half of its content was found to be $10.04 \mathrm{~h}$ following oral administration of GLY.

In vivo pharmacokinetic study of GLY-8 formulation in 6 rats following single oral administration of $1 \mathrm{mg} / \mathrm{kg}$ b.w. of GLY was performed. The concentration of drug in the plasma samples was obtained from the blood samples which were collected from each rat throughout a period of $12 \mathrm{~h}$ and were calculated. Fig. 14 shows the representative chromatogram of GLY from rat plasma. The concentration-time profile of GLY in the rat plasma is shown in Table 10. The mean estimated non-compartmental pharmacokinetic parameters derived from the plasma concentration profiles are summarized in Table 10, and Fig. 15 shows the mean plasma concentrations of GLY across the time points following per oral administration. GLY plasma concentrations calculated at different time intervals for 6 rats showed that the drug was readily absorbed after oral administration and the peak plasma concentration of GLY was reached by $2 \pm 1.01 \mathrm{~h}$. The $\mathrm{C}_{\max }$ of GLY was

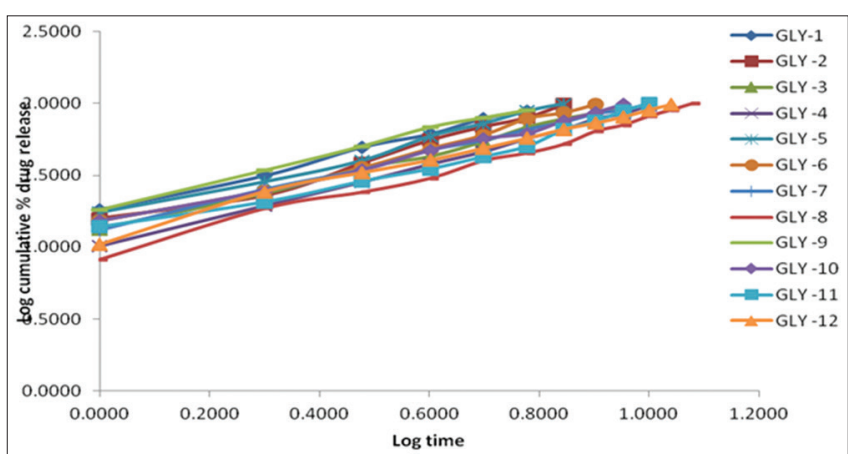

Fig. 10: Korsmeyer-Peppas plot

$0.604 \pm 0.03$. The values of AUC ${ }_{(0-t)}$ and AUC ${ }_{(0-\infty)}$ were found to be $2.562 \pm 0.41$ and $2.147 \pm 0.45 \mu \mathrm{g} . \mathrm{h} / \mathrm{ml}$, respectively. Till date, an extensive study of GLY pharmacokinetic parameters such as its distribution pattern, mechanism of metabolism, and excretion was not reported and still under investigation.

\section{DISCUSSION}

In the current work, an attempt has been made to introduce a new drug delivery system "nano particulates incorporated in suspension." The mechanism of drug release from the nanosuspension release was explained in four steps. They are (1) Formulation of colloidal system, (2) size reduction to nanoparticles through homogenization, (3) counter diffusion mechanism of nano-sized particles into a suspension, and (4) sustained release of the drug and particle detachment.

Drug and excipient compatibility studies were performed using FTIR spectrophotometer and DSC, and no marked incompatibilities were found. GLY has been scanned in the UV spectrophotometer and found $\lambda_{\max }$ at $229 \mathrm{~nm}$. A CC was constructed in the concentration range of $25-150 \mu \mathrm{g} / \mathrm{ml}$. The curve was the best fit with $\mathrm{R}^{2}$ value of 0.9983 and slope of 0.0056 . The FTIR spectra for pure drug of GLY and with blend are shown in Figs. 1 and 2. Furthermore, the GLY compatibility with polymer blend was proved by DSC. The DSC thermogram of GLY (Fig. 6) 


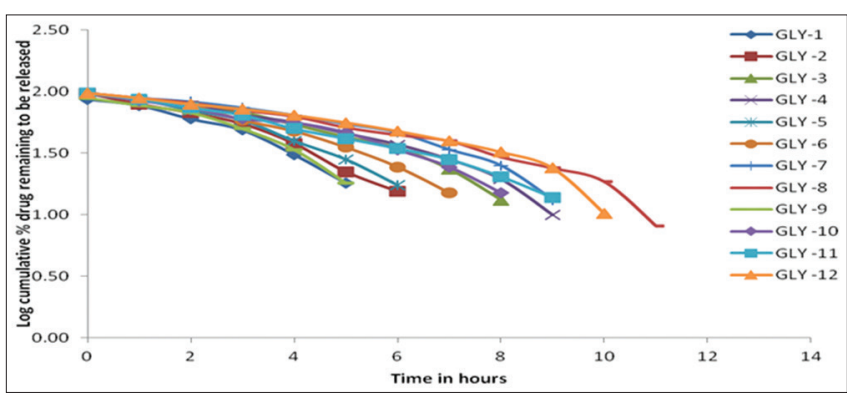

Fig. 11: First-order plot

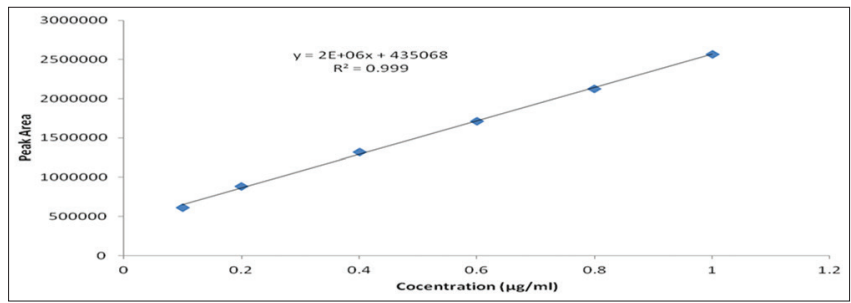

Fig. 12: Calibration curve of glyburide in rat plasma



Fig. 13: Blank chromatogram from rat plasma sample

reveals the melting point at $168.7^{\circ} \mathrm{C}$, whereas GLY with blend (Figs. 6 and 7) also shows the melting point at $168.0^{\circ} \mathrm{C}$ which proved that drug had good thermal stability. Nanosuspension formulation of GLY was prepared based on the principle of quasi emulsification solvent diffusion method. With increase in concentration of polymers, the prepared nanosuspension formulations had shown good correlation with size and particle charge (PVP, HPMC, and EDG RL 100). As the concentration of polymer increased, an increase in the entrapment efficiency was observed and average particle size also increased. GLY-8 was the best formulation with a particle size of $85-96 \mathrm{~nm}, 93.53 \%$ drug entrapment, and $90.26 \pm 1 \mathrm{mV}$ of zeta potential. This formulation showed percentage drug release of $99.85 \%$ in $24 \mathrm{~h}$. The release exponents showed that value of " $\mathrm{n}$ " was $0.975513(<1)$ indicating non-Fickian transport mechanism on drug release behavior. The drug relapse followed zero order with regression value near to 0.99 and diffusion type of release from the Higuchi equation regression value of 0.97 .

In vivo pharmacokinetic study for optimized formulation (GLY-8) was carried out using the developed and validated reverse-phase highperformance liquid chromatography method on Wistar albino rats. For GLY-8, $\mathrm{C}_{\max }$ and $\mathrm{T}_{\text {max }}$ were found to be $0.604 \pm 0.03 \mu \mathrm{g} / \mathrm{ml}$ and $2 \pm 1.01 \mathrm{~h}$, respectively. The values of $\mathrm{t}_{1 / 2}(\mathrm{~h}), \mathrm{AUC}_{(0-\mathrm{t})}$, and $\mathrm{AUC}_{(0-\infty)}$ were found to be $10.04 \mathrm{~h}, 2.562 \pm 0.41 \mu \mathrm{g} . \mathrm{h} / \mathrm{ml}$, and $2.147 \pm 0.45 \mu \mathrm{g} . \mathrm{h} / \mathrm{ml}$ respectively. Based on the obtained results, oral administration of nanosuspension could not only provide the better absorption of poorly water-soluble drugs but may also reduce toxicity and provide a new tool in drug delivery system.

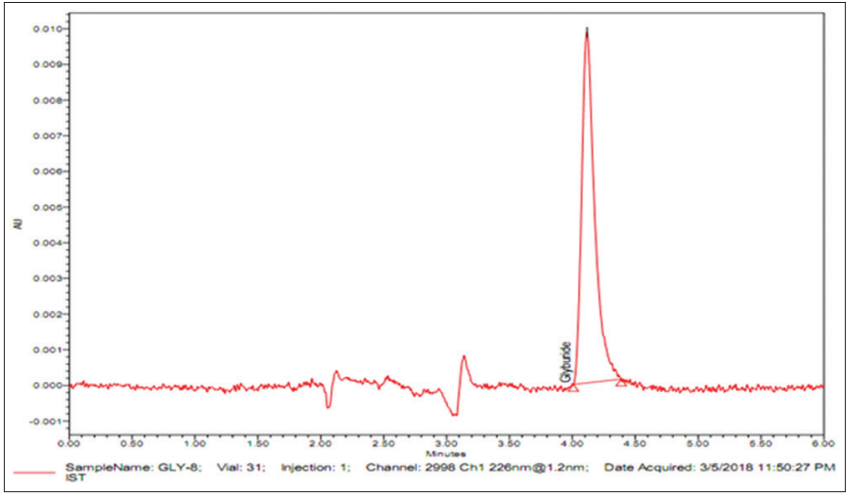

Fig. 14: Blank and $\mathrm{C}_{\max }$ chromatogram of glyburide from rat plasma sample

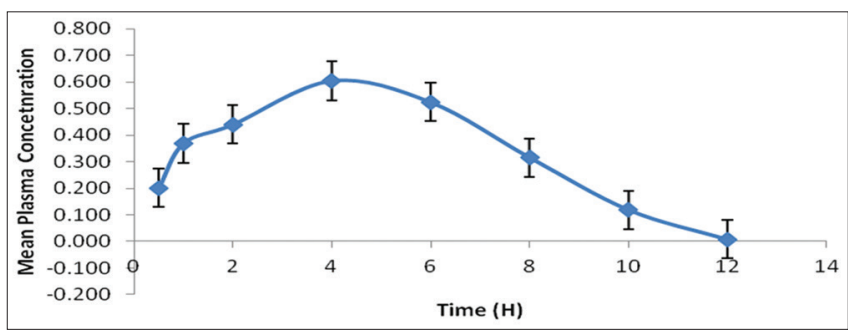

Fig. 15: Mean plasma concentration of glyburide in rat plasma

\section{CONCLUSION}

GLY is poorly water-soluble (BCS class II) drug. The poor aqueous solubility of this drug gives rise to many obstacles in the design of pharmaceutical formulations and leads to variable oral bioavailability. Hence, this drug was selected for the present study for enhancing their rate of dissolution and oral bioavailability through nanosuspension technologies. Nanosuspensions were prepared and evaluated by in vitro and in vivo methods. From the obtained results, it was concluded that all the nanosuspensions prepared were found to be fine and nano range. GLY was taken in phosphate buffer with a pH 7.4 at $229 \mathrm{~nm}$, respectively, and nanosuspensions were prepared. The obtained nanosuspensions were checked for the characterization of size analysis, drug content, entrapment efficiency, drug release studies in in vitro, FTIR spectroscopy studies, DSC studies, and SEM studies. FTIR spectra showed no interactions between excipients and drug of the nanosuspension. Among all GLY nanosuspension formulations, GLY-8 formulation was considered as an optimized formulation which showed maximum drug release, i.e., $99.85 \%$ in $24 \mathrm{~h}$.

\section{CONFLICTS OF INTEREST}

There were no conflicts of interest.

\section{AUTHORS' CONTRIBUTION}

We declare that this work was done by the authors named in this article and all liabilities pertaining to claims relating to the content of this article will be borne by the authors.

Mr. Pradeep Kumar M collected the data, analyzed the data, performed all the laboratory works, and wrote the introduction, discussion, and the material and method part.

Dr. K. B. Chandra Sekhar, supervisor of this study, helped in selection of the topic, procuring chemicals, and drugs from different laboratories and also helped me a lot in drafting the manuscript and proofreading. 


\section{ACKNOWLEDGMENT}

The author is thankful to guide Dr. KB Chandra Sekhar for his tremendous support and constant encouragement to complete this research work.

The author is also thankful to the management and principal of Vasavi Institute of Pharmaceutical Sciences for providing all facilities for this research work.

\section{REFERENCES}

1. Bergstroem CA, Wassvik CM, Johansson K, Hubatsch I. Poorly soluble marketed drugs display solvation limited solubility. J Med Chem 2007;50:5858-62.

2. Wei H, Dalton C, Di Maso M, Kanfer I, Löbenberg R. Physicochemical characterization of five glyburide powders: A BCS based approach to predict oral absorption. Eur J Pharm Biopharm 2008;69:1046-56.

3. Kohli S, Pal A, Jain S. Preparation, characterization and evaluation of poly (lactide-co-glycolide) microspheres for the controlled release of zidovudine. Int J Pharm Pharm Sci 2017;9:70-7.

4. Reza MI, Goel D, Gupta RK, Warsi MH. Formulation of ketoconazole loaded nano dispersive gel using swollen micelles technique and its in vitro characterization. Int J Pharm Pharm Sci 2017;10:162-6.

5. Atar SA, Soniwala M. Formulation and evaluation of pectin-calcium chloride beads of azathioprine for colon targeted drug delivery system. Int J Pharm Pharm Sci 2018;10:172-7.

6. Powar PV. Development status in the meadow of nanostructure magnetic drug delivery system and its promising applications. Int $\mathrm{J}$
Pharm Pharm Sci 2017;9:10-7.

7. Kunal S, Ravindra B. UV spectrophotometric method for the estimation of azilsartan medoxomil in bulk and pharmaceutical formulations. World J Pharm Res 2015;4:1667-72.

8. Gandhi S, Mittal P, Pahade A, Rege S. Development and validation of stability indicating HPTLC method for estimation of azilsartan medoxomil. Pharm Sci Monit 2015;6:224-32.

9. Winarti L, Sari LO, Nugroho AE. Naringenin-loaded chitosan nanoparticles formulation and its in vitro evaluation against $447 \mathrm{~d}$ breast cancer cell line. Indones J Pharm 2015;26:145-57.

10. Pignatello R, Bucolo C, Ferrara P, Maltese A, Puleo A, Puglisi G, et al. Eudragit RS100 nanosuspensions for the ophthalmic controlled delivery of ibuprofen. Eur J Pharm Sci 2002;16:53-61.

11. Prakash S, Vidyadhara S, Sasidhar RL, Abhijit D, Akhilesh D. Development and characterization of ritonavir nanosuspension for oral use. Pharm Lett 2013;5:48-55.

12. Dong Y, Ng WK, Hu J, Shen S, Tan RB. A continuous and highly effective static mixing process for antisolvent precipitation of nanoparticles of poorly water-soluble drugs. Int J Pharm 2010;386:256-61

13. Huong DT, Takahashi Y, Ide T. Activity and mRNA levels of enzymes involved in hepatic fatty acid oxidation in mice fed citrus flavonoids. Nutrition 2006;22:546-52.

14. Rajalakshmi R, Venkataramudu T, Kumar R, Divya K, Kiranmayi M. Design and characterization of valsartan nanosuspension. Int $\mathrm{J}$ Pharmacother 2012;2:70-81.

15. Kroyer $\mathrm{G}$. The antioxidant activity of citrus fruit peels. Z Ernahrungswiss 1986;25:63-9.

16. Sahu BP, Das MK. Nanosuspension for enhancement of oral bioavailability of felodipine. Appl Nanosci 2013;4:1-9. 